\title{
Compound System Reliability Test Design Based on Bayesian Approach and Monte Carlo Method
}

\author{
Anyang Zhang ${ }^{1, a}$ and Qi Liü,b \\ ${ }^{1,2}$ College of Informational Systems and Management, National University of Defense Technology, \\ Changsha, P. R. China \\ asuper_879@163.com
}

Keywords: Reliability test design; Information fusion; CSRTD; Bayesian approach; Monte Carlo method.

\begin{abstract}
Analyzing the current lack of reliability test design and considering the producer's risk and consumer's risk, the reliability test design model for compound system (CSRTD) based on information fusion was constructed. Analyzing the requirements and constraint conditions of reliability test design, the information fusion technology between binomial distributed subsystems and their system was presented by means of Bayesian approach and Monte Carlo method. According to posteriori risk criteria, the formulas of producer's risk and consumer's risk were deduced. The solution of computing the optimum test plan was given by Matlab software. Finally, an example of missile launching installation was given to demonstrate the validity of the model.
\end{abstract}

\section{Introduction}

In the field of reliability test, the evaluation of the system reliability of product with high reliability and long life is one of the important problems, especially for the complex system such as aircrafts, missiles and satellites. Therefore, due to high cost and long test time, it is improper to perform reliability test by the traditional test method [1-3].

As the Bayesian theory has the advantage on reduce actual test numbers, it has been widely studied in the field of reliability test in recent years and gradually recognized by the international academic community. Belkacem et al [4] used the Bayesian network approach to formulate the reliability model of systems with uncertain structures. Sanku [5] obtained Bayes' estimators for the unknown parameter of an Inverse Rayleigh distribution under symmetric and asymmetric linear exponential loss functions using a non-informative prior. Cai et al [6] developed a redundant software system for subsea blowout preventers by Bayesian networks.

However, in many circumstance, the application of these methods may not give satisfactory result. The existing methods have at least the following problem:

- When encountering complex compound system, we cannot obtain the prior distribution of system from the subsystems by analytic method, so the posterior distribution of system cannot be deduced by Bayesian theory.

- The subsystems' and system's test cost should be totally taken into consideration, but the current researches pay fewer attentions on the total cost of reliability test.

According to the above shortcomings, this paper presents a subsystem-to-system information fusion method for compound system by means of Bayesian approach and Monte Carlo method. And CSRTD (the reliability test design model for compound system) is built to figure out the minimum cost of the compound system when considering the constraints. At last, one numerical example is used to demonstrate the validity of the model.

\section{CSRTD Description}

The Basic Assumptions

In order to construct the model precisely, five assumptions are present as following.

1) The assumption of model 
Supposing that a compound system is made up of $m$ subsystems, the reliability parameter of each subsystem is $R_{i}(i=1,2, \cdots, m)$ and $R_{m+1}$ denotes the compound system's. In this paper, the variables whose subscript is $m+1$ describe the characteristic of the compound system.

If all the failures are independent, then the compound system structure function is $R_{m+1}=g\left(R_{1}, R_{2}, \cdots, R_{m}\right)$.

2) The assumption of prior information

Prior information is people's realization of unknown parameters before performing reliability tests. In this paper, all the subsystems' and system's test results are success/failure data, and can be analyzed by binomial distribution. Therefore, supposing all the subsystems and system are binomial system, the prior distribution of $R_{i}(i=1,2, \cdots, m+1)$ is beta distribution $B\left(\alpha_{i}, \beta_{i}\right)$, where. $\alpha_{i}$.and $\beta_{i}$ are hyper-parameters. $\pi\left(R_{i}\right)(i=1,2, \cdots, m)$ denotes the prior distribution of..th subsystem and $\pi^{(2)}\left(R_{m+1}\right)$ denotes the compound system's. Another prior distribution $\pi^{(1)}\left(R_{m+1}\right)$ of the compound system is derived from the subsystems by information fusion technology.

3) The assumption of reliability parameter

In the reliability demonstration test, the consumer requires a desired value $R_{0}$ and a lowest accepted value $R_{1}$ of the system's reliability, which is equivalent to test the following statistical hypothesis

$H_{0}: R_{m+1} \geq R_{0} \quad H_{1}: R_{m+1}<R_{1}$.

4) The assumption of test cost

During system reliability demonstration test, we suppose that the testing cost is a linear function of the actual test number of the subsystems and system, i.e.

$C_{T}=\sum_{i=1}^{m+1} C_{i}^{I}+\sum_{i=1}^{m+1} C_{i}^{U} n_{i}+C_{m+1}^{\alpha} \alpha\left(n_{m+1}, f_{m+1}\right)+C_{m+1}^{\beta} \beta\left(n_{m+1}, f_{m+1}\right)$.

Where

$C_{T}$ - Total reliability test cost.

$C_{i}^{I}-i$ th subsystem's (system's) initial reliability test cost, for $i=1,2, \cdots, m+1$. The initial reliability test cost includes all the cost which is necessary to start the reliability test, such as cost of test facility construction, staff training fee, test prepare fee, etc.

$C_{i}^{U}-i$ th subsystem's (system's) actual unit reliability test cost, for $i=1,2, \cdots, m+1$. The unit test cost includes all the cost which is necessary to operate the actual reliability test, such as staffs' salary, subsystem's (system's) cost price, facility charge for use, etc.

$n_{i}-i$ th subsystem's (system's) actual reliability test number, for $i=1,2, \cdots, m+1$.

$f_{m+1}$-System's reliability failure test number.

$\alpha\left(n_{m+1}, f_{m+1}\right)$-Producer's risk of system.

$\beta\left(n_{m+1}, f_{m+1}\right)$-Consumer's risk of system.

$C_{m+1}^{\alpha}$-System's reliability test cost which producer's risk leads to, when rejecting $H_{0}$.

$C_{m+1}^{\beta}$-System's reliability test cost which consumer's risk leads to, when rejecting $H_{1}$.

5) The assumption of risks

In this article, the posterior risk criteria is used to calculate the produce's risk and the consumer's risk [7]. According to the posterior risk criteria, the definition of the two types of risks is

Posterior Producer's Risk $=P\left(H_{0} \mid\right.$ Test is Failed $)$

Posterior Consumer's Risk $=P\left(H_{1} \mid\right.$ Test is Passed $)$

According to equation (4), $\alpha\left(n_{m+1}, f_{m+1}\right)$ and $\beta\left(n_{m+1}, f_{m+1}\right)$ can be deduced, i.e.

$\alpha\left(n_{m+1}, f_{m+1}\right)=\frac{\int_{\Theta_{0}} P\left(f>f_{m+1} \mid R_{m+1}\right) \pi\left(R_{m+1} \mid \alpha_{m+1}^{*}, \beta_{m+1}^{*}\right) d R_{m+1}}{\int_{\Theta} P\left(f>f_{m+1} \mid R_{m+1}\right) \pi\left(R_{m+1} \mid \alpha_{m+1}^{*}, \beta_{m+1}^{*}\right) d R_{m+1}}$. 
$\beta\left(n_{m+1}, f_{m+1}\right)=\frac{\int_{\Theta_{1}} P\left(f \leq f_{m+1} \mid R_{m+1}\right) \pi\left(R_{m+1} \mid \alpha_{m+1}^{*}, \beta_{m+1}^{*}\right) d R_{m+1}}{\int_{\Theta} P\left(f \leq f_{m+1} \mid R_{m+1}\right) \pi\left(R_{m+1} \mid \alpha_{m+1}^{*}, \beta_{m+1}^{*}\right) d R_{m+1}}$.

Where

$\left(\alpha_{m+1}^{*}, \beta_{m+1}^{*}\right)$-The two parameters of system posterior distribution after information fusion.

\section{Construction of CSRTD}

Based on the Section 2.1 and the minimization of total test cost, CSRTD can be built as

$\operatorname{Min}\left(C_{T}\right)=\operatorname{Min}\left[\sum_{i=1}^{m+1} C_{i}^{I}+\sum_{i=1}^{m+1} C_{i}^{U} n_{i}+C_{m+1}^{\alpha} \alpha\left(n_{m+1}, f_{m+1}\right)+C_{m+1}^{\beta} \beta\left(n_{m+1}, f_{m+1}\right)\right]$.

St

$\left\{\begin{array}{l}C_{i}^{I}>0, C_{i}^{U}>0, i=1,2, \cdots, m+1 \\ C_{m+1}^{\alpha}>0, C_{m+1}^{\beta}>0 \\ N_{i}^{L} \leq n_{i} \leq N_{i}^{U}, i=1,2, \cdots, m+1 \\ \alpha\left(n_{m+1}, f_{m+1}\right) \leq \alpha_{0} \\ \beta\left(n_{m+1}, f_{m+1}\right) \leq \beta_{0}\end{array}\right.$.

Where

$N_{i}^{U}$-The maximum test numbers that can be provided by the producer and consumer.

$N_{i}^{L}$ - The minimum test numbers that can be accepted by the producer and consumer.

$\alpha_{0}$-The significance level of producer's risk.

$\beta_{0}$-The significance level of consumer's risk.

\section{Information Fusion and Solution of CSRTD}

In this article, we focus on two ways of information fusion. The first is how the information of subsystems is fused into the system's, and the second is how to combine the system prior information $\pi^{(1)}\left(R_{m+1}\right)$ from subsystems with the system inherent prior information $\pi^{(2)}\left(R_{m+1}\right)$.

1) Solution of the first way

According to Bayesian theory and the assumption of prior information, the posterior distribution of $R_{i}(i=1,2, \cdots, m)$ can be calculated, i.e.

$\pi\left(R_{i} \mid\left(n_{i}, f_{i}\right)\right)=\frac{\pi\left(R_{i} \mid \alpha_{i}, \beta_{i}\right) R_{i}^{n_{i}-f_{i}}\left(1-R_{i}\right)^{f_{i}}}{\int_{0}^{1} \pi\left(R_{i} \mid \alpha_{i}, \beta_{i}\right) R_{i}^{n_{i}-f_{i}}\left(1-R_{i}\right)^{f_{i}} d R_{i}}=\pi\left(R_{i} \mid \alpha_{i}+n_{i}-f_{i}, \beta_{i}+f_{i}\right)$.

So the posterior distribution of $R_{i}$ is $B\left(\alpha_{i}+n_{i}-f_{i}, \beta_{i}+f_{i}\right)$.

To complex compound system, it's difficult to figure out the analytical solution of its prior information $\pi^{(1)}\left(R_{m+1}\right)$. Therefore, the paper uses Monte Carlo analysis to obtain the estimation of system prior information, and then the distribution $\pi^{(1)}\left(R_{m+1}\right)$ can be given by the goodness of fit. The steps of Monte Carlo (SMC) are as follow.

Step 1: Set the value of simulation times $N$, and set $k=1$;

Step 2: By Matlab, we can calculate the reliability simulation value of each subsystem respectively according to $\pi\left(R_{i} \mid \alpha_{i}+n_{i}-f_{i}, \beta_{i}+f_{i}\right)$, i.e.

$T_{1}^{(k)}, T_{2}^{(k)}, \cdots, T_{m}^{(k)}$.

Step 3: According to the equation(1), the reliability simulation value of system is $T_{m+1}^{(k)}=g\left(T_{1}^{(k)}, T_{2}^{(k)}, \cdots, T_{m}^{(k)}\right)$. 
Step 4: Judge: if $k<N$, then $k=k+1$ and move to Step 2.

Step 5: To $\left\{T_{m+1}^{(k)}\right\}(k=1,2, \cdots, N), \pi^{(1)}\left(R_{m+1}\right)$ can be figured out by fitting of distribution and parameter estimation.

2) Solution of the second way

Weighting fusion algorithm [8] is used to solve the fusion of system prior distribution. Supposing that the source of prior distribution and the accuracy of prior distribution are fully analyzed, we can obtain the weight values $W_{1}, W_{2}$, for $W_{1}+W_{2}=1$. Therefore, the system prior distribution is $\pi\left(R_{m+1}\right)=W_{1} \pi^{(1)}\left(R_{m+1}\right)+W_{2} \pi^{(2)}\left(R_{m+1}\right)$.

So, the posterior distribution of $R_{m+1}$ is

$$
\pi\left(R_{m+1} \mid\left(n_{m+1}, f_{m+1}\right)\right)=\frac{\pi\left(R_{m+1}\right) L\left(R_{m+1} \mid\left(n_{m+1}, f_{m+1}\right)\right)}{\int_{0}^{1} \pi\left(R_{m+1}\right) L\left(R_{m+1} \mid\left(n_{m+1}, f_{m+1}\right)\right) d R_{m+1}} .
$$

Where

$$
L\left(R_{m+1} \mid\left(n_{m+1}, f_{m+1}\right)\right) \text {-The likelihood function of } R_{m+1} .
$$

According to equation (12) and (13), then

$$
\pi\left(R_{m+1} \mid\left(n_{m+1}, f_{m+1}\right)\right)=\frac{1}{m\left(\left(n_{m+1}, f_{m+1}\right) \mid \pi\right)}\left[W_{1} \pi^{(1)}\left(R_{m+1}\right)+W_{2} \pi^{(2)}\left(R_{m+1}\right)\right] L\left(R_{m+1} \mid\left(n_{m+1}, f_{m+1}\right)\right) \text {. }
$$

Where

$$
\begin{gathered}
m\left(\left(n_{m+1}, f_{m+1}\right) \mid \pi\right) \text {-The marginal density of } R_{m+1}, \\
m\left(\left(n_{m+1}, f_{m+1}\right) \mid \pi\right)=\int_{0}^{1} \pi\left(R_{m+1}\right) L\left(R_{m+1} \mid\left(n_{m+1}, f_{m+1}\right)\right) d R_{m+1} .
\end{gathered}
$$

To $h$ th prior distribution, its posterior distribution can be calculated, i.e.

$$
\pi^{(h)}\left(R_{m+1} \mid\left(n_{m+1}, f_{m+1}\right)\right)=\frac{\pi^{(h)}\left(R_{m+1}\right) L\left(R_{m+1} \mid\left(n_{m+1}, f_{m+1}\right)\right)}{\int_{0}^{1} \pi^{(h)}\left(R_{m+1}\right) L\left(R_{m+1} \mid\left(n_{m+1}, f_{m+1}\right)\right) d R_{m+1}}(h=1,2) .
$$

According to equation (14), define

$$
\begin{aligned}
& m\left(\left(n_{m+1}, f_{m+1}\right) \mid \pi^{(h)}\right)=\int_{0}^{1} \pi^{(h)}\left(R_{m+1}\right) L\left(R_{m+1} \mid\left(n_{m+1}, f_{m+1}\right)\right) d R_{m+1} . \\
& \lambda_{i}\left(n_{m+1}, f_{m+1}\right)=\frac{W_{i} m\left(\left(n_{m+1}, f_{m+1}\right) \mid \pi^{(h)}\right)}{\sum_{h=1}^{2} W_{h} m\left(\left(n_{m+1}, f_{m+1}\right) \mid \pi^{(h)}\right)}(i=1,2) .
\end{aligned}
$$

According to equation (13), (15), (16) and (17), the posterior distribution of $R_{m+1}$ is

$$
\pi\left(R_{m+1} \mid\left(n_{m+1}, f_{m+1}\right)\right)=\sum_{h=1}^{2} \lambda_{h}\left(n_{m+1}, f_{m+1}\right) \pi^{(h)}\left(R_{m+1} \mid\left(n_{m+1}, f_{m+1}\right)\right) \text {. }
$$

We can see that the system posterior distribution is the weighting sum of the posterior distribution from different prior information source.

To solve the CSRTD, it requires calculating the equation (5) and (6) first. We need to know actual subsystem's (system's) test results $\left(n_{i}, f_{i}\right)(i=1,2, \cdots, m+1)$, but in test design stage, the actual test numbers $n_{i}$ and failure test numbers $f_{i}(i=1,2, \cdots, m+1)$ are unknown, and need to be solved. It is difficult to find the solution of $n_{1}, n_{2}, \cdots, n_{m+1}$ for all possible $f_{1}, f_{2}, \cdots, f_{m+1}$. So, in practice, the failure test numbers can be fixed, and for given $f_{1}, f_{2}, \cdots, f_{m+1}$, to find the proper actual test numbers $n_{1}, n_{2}, \cdots, n_{m+1}$. Nowadays, the subsystems and system are usually very reliable and their reliability are usually very high, especially for the systems which has passed the reliability growth test and environment stress test. So, the failure test numbers can be fixed at 0 or other number which is much less than the actual test number. The equation (5) and (6) can be deduced further as follow, when $f_{m+1}=1$. 


$$
\begin{aligned}
& \alpha\left(n_{m+1}, 1\right)=\frac{\int_{R_{0}}^{1} \sum_{f=2}^{n_{m+1}}\left(\begin{array}{l}
n_{m+1} \\
f
\end{array}\right)\left(1-R_{m+1}\right)^{f+\beta_{m+1}^{*}-1} R_{m+1}^{\alpha_{m+1}^{*}+n_{m+1}-f-1} d R_{m+1}}{\int_{0}^{1} \sum_{f=2}^{n_{m+1}}\left(\begin{array}{l}
n_{m+1} \\
f
\end{array}\right)\left(1-R_{m+1}\right)^{f+\beta_{m+1}^{*}-1} R_{m+1}^{\alpha_{m+1}^{*}+n_{m+1}-f-1} d R_{m+1}} . \\
& \beta\left(n_{m+1}, 1\right)=\frac{\int_{0}^{R_{1}} \sum_{f=0}^{1}\left(\begin{array}{l}
n_{m+1} \\
f
\end{array}\right)\left(1-R_{m+1}\right)^{f+\beta_{m+1}^{*}-1} R_{m+1}^{\alpha_{m+1}^{*}+n_{m+1}-f-1} d R_{m+1}}{\int_{0}^{1} \sum_{f=0}^{1}\left(\begin{array}{l}
n_{m+1} \\
f
\end{array}\right)\left(1-R_{m+1}\right)^{f+\beta_{m+1}^{*}-1} R_{m+1}^{\alpha_{m+1}^{*}+n_{m+1}-f-1} d R_{m+1}} .
\end{aligned}
$$

Because the calculation includes the integral operation of $\alpha\left(n_{m+1}, f_{m+1}\right)$ and $\beta\left(n_{m+1}, f_{m+1}\right)$, it is difficult to solve the CSRTD (equation (7) and (8)) by analytical method. So in this paper, the numerical method is suggested to solve the CSRTD, such as using Matlab. The steps are as follows. Step 1: According to test environment, set the values of $C_{i}^{I}, C_{i}^{U}, C_{m+1}^{\alpha}, C_{m+1}^{\beta}, N_{i}^{L}, N_{i}^{U}, \alpha_{0}, \beta_{0}, R_{0}, R_{1}, f_{i}$, for $i=1,2, \cdots, m+1$. Set the system prior distribution $\pi^{(2)}\left(R_{m+1}\right)$ and the weighting values $W_{1}, W_{2}$.

Step 2: According to the prior information of subsystems, such as historic test results, expert's information, confirm the subsystems' prior distributions $\pi\left(R_{i}\right)(i=1,2, \cdots, m)$.

Step 3: According to equation (9), figure out the subsystems' posterior distributions $\pi\left(R_{i} \mid\left(n_{i}, f_{i}\right)\right)$. Using SMC method (in Chapter 3), we can obtain one of system's prior distribution $\pi^{(1)}\left(R_{m+1}\right)$.

Step 4: According to equation (16), (17) and (18), we can obtain the system's posterior distribution $\pi\left(R_{m+1} \mid\left(n_{i}, f_{i}\right)\right)$.

Step 5: Set the constrain condition of actual test numbers $n_{i}$, denote the lower limit as $N_{i}^{L}$, upper limit as $N_{i}^{U}$, for $i=1,2, \cdots, m+1$. To test plan, $N_{i}^{L}=1, N_{i}^{U}=N_{i}$, for $i=1,2, \cdots, m+1$. Definite a data set $\left(n_{1}, n_{2}, \cdots, n_{m+1}\right)$, where $n_{i}$ denote the actual test numbers of subsystem (system) $i$, for $i=1,2, \cdots, m+1$. List all the possible value of $\left(n_{1}, n_{2}, \cdots, n_{m+1}\right)$, denote the result as a set $S$, then $S=\left\{\left(N_{1}^{L}, N_{2}^{L}, \cdots, N_{m+1}^{L}\right),\left(N_{1}^{L}, N_{2}^{L}, \cdots, N_{m+1}^{L}+1\right), \cdots,\left(N_{1}^{U}, N_{2}^{U}, \cdots, N_{m+1}^{U}\right)\right\}$. Denote the $k$ th element in set $S$ as $S(k)$, for $k=1,2, \cdots, \prod_{i=1}^{m+1}\left(N_{i}^{U}-N_{i}^{L}+1\right)$.

Step 6: Set $k=1$, let the initial test costs $C=\inf$.

Step 7: Judge: if $k \leq \prod_{i=1}^{m+1}\left(N_{i}^{U}-N_{i}^{L}+1\right)$, then move to Step 8, else move to Step 11.

Step 8: To $S(k)$, according to the equation (5) and (6), calculate $\alpha\left(n_{m+1}, f_{m+1}\right)$ and $\beta\left(n_{m+1}, f_{m+1}\right)$.

Step 9: If $\alpha\left(n_{m+1}, f_{m+1}\right) \leq \alpha_{0}$ and , then move to Step 10, else let $k=k+1$, move to Step 7;

Step 10: According to the equation (3), calculate the test $\operatorname{cost} C_{T}$, and judge

- If $C_{T} \geq C$, let $k=k+1$, move to Step 7 .

- If $C_{T}<C, S(k)$ will be the current optimum actual test combination, denote the optimum test plan as $\left(\left(n_{1}^{O}, f_{1}\right), \cdots,\left(n_{i}^{O}, f_{i}\right), \cdots,\left(n_{m+1}^{O}, f_{m+1}\right)\right)$, where

$S(k)=\left(\left(n_{1}^{O}, f_{1}\right), \cdots,\left(n_{i}^{O}, f_{i}\right), \cdots,\left(n_{m+1}^{O}, f_{m+1}\right)\right)$. Let $C=C_{T}, k=k+1$, move to Step 7.

Step 11: End calculation.

\section{Numerical Example Analysis}

A missile launching installation consists of one target channel (subsystem 1), two missile channels (subsystem 2 and subsystem 3), and four launching installations (subsystems 4-7). The missile 
launching installation is a compound system as shown in Figure 1, and every subsystem reliability test results are binomial distributed and independent.

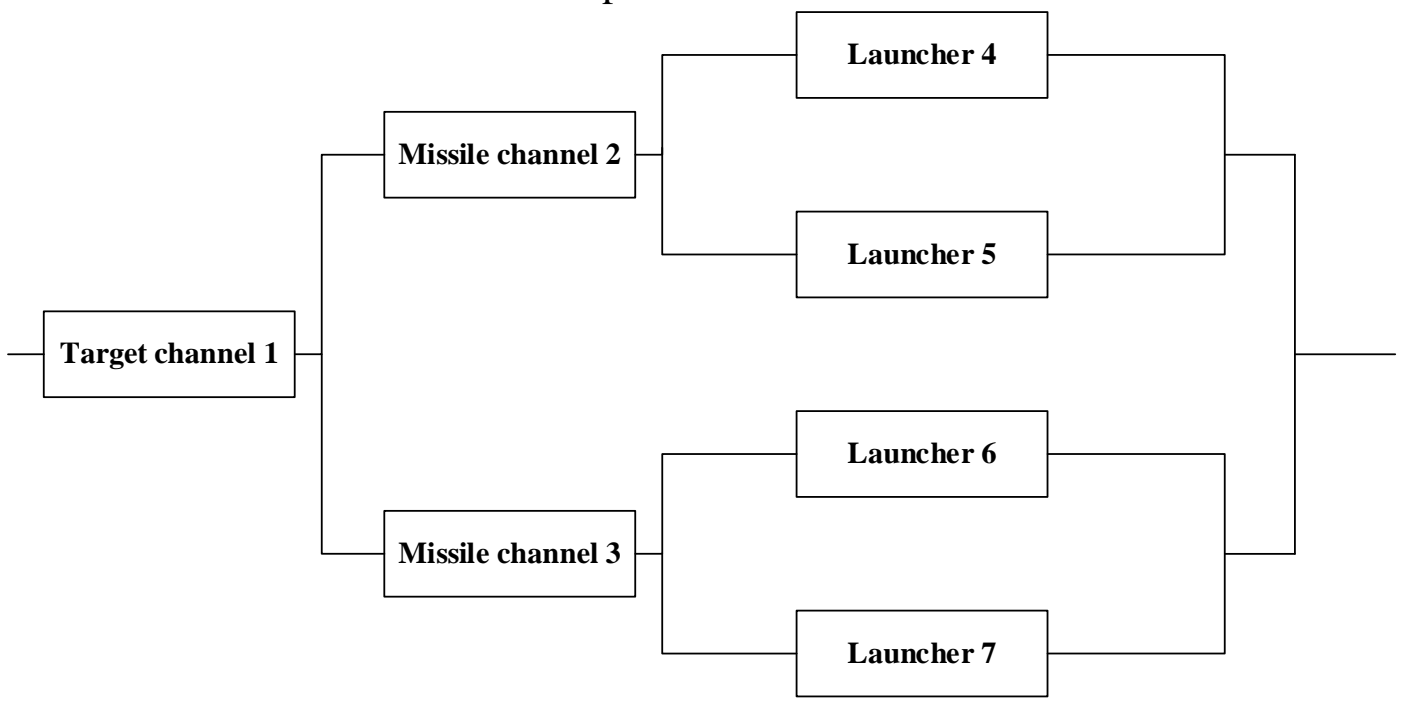

Figure 1 The reliability block diagram of the missile launching installation

Table 1 Parameter value assignment

\begin{tabular}{cc}
\hline Parameter & Value \\
\hline$C^{I}$ & $(15,10,10,20,20,20,20,100)$ \\
$C^{U}$ & $(30,15,15,50,50,50,50,100)$ \\
$N^{L}$ & $(1,1,1,1,1,1,1,1)$ \\
$N^{U}$ & $(8,11,11,7,6,7,8,3)$ \\
$\left(C_{8}^{\alpha}, C_{8}^{\beta}\right)$ & $(0.1,0.1)$ \\
$\left(\alpha_{0}, \beta_{0}\right)$ & $(0.98,0.9)$ \\
$\left(R_{0}, R_{1}\right)$ & $(0,0,0,0,0,0,0,0)$ \\
$f$ &
\end{tabular}

According to the steps of algorithm, Table 2 lists parts of the possible test plan. The minimum of $k$ is 1 , and the maximum is $\prod_{i=1}^{8}\left(N_{i}^{U}-N_{i}^{L}+1\right)=6830208$.

Table 2 Parts of the calculation results

\begin{tabular}{|c|c|c|c|c|c|c|c|c|c|c|c|}
\hline$k$ & $n_{1}$ & $n_{2}$ & $n_{3}$ & $n_{4}$ & $n_{5}$ & $n_{6}$ & $n_{7}$ & $n_{8}$ & $\alpha$ & $\beta$ & $C_{T}$ \\
\hline 1 & 1 & 1 & 1 & 1 & 1 & 1 & 1 & 1 & 0.1058 & $2.46 \times 10^{-20}$ & 998.2 \\
\hline 218 & 1 & 1 & 1 & 1 & 2 & 3 & 1 & 2 & 0.1105 & $3.34 \times 10^{-20}$ & 1267 \\
\hline 968386 & 2 & 2 & 6 & 2 & 5 & 2 & 4 & 1 & 0.1050 & $3.42 \times 10^{-20}$ & 1565 \\
\hline 968717 & 2 & 2 & 6 & 3 & 1 & 2 & 2 & 2 & 0.0993 & $1.60 \times 10^{-20}$ & 1392.3 \\
\hline 968719 & 2 & 2 & 6 & 3 & 1 & 2 & 3 & 1 & 0.1040 & $8.68 \times 10^{-21}$ & 1361 \\
\hline 968722 & 2 & 2 & 6 & 3 & 1 & 2 & 4 & 1 & 0.1022 & $5.63 \times 10^{-21}$ & 1403.8 \\
\hline 968725 & 2 & 2 & 6 & 3 & 1 & 2 & 5 & 1 & 0.1031 & $9.87 \times 10^{-21}$ & 1457.4 \\
\hline 3255712 & 4 & 9 & 11 & 3 & 6 & 2 & 6 & 1 & 0.1062 & $2.70 \times 10^{-20}$ & 2009.8 \\
\hline 3255715 & 4 & 9 & 11 & 3 & 6 & 2 & 7 & 1 & 0.1080 & $1.11 \times 10^{-20}$ & 2067 \\
\hline 3835582 & 5 & 6 & 5 & 5 & 1 & 6 & 8 & 1 & 0.0995 & $1.53 \times 10^{-20}$ & 2028 \\
\hline 6765553 & 8 & 11 & 2 & 6 & 6 & 2 & 1 & 1 & 0.0985 & $5.72 \times 10^{-21}$ & 1894 \\
\hline 6830208 & 8 & 11 & 11 & 7 & 6 & 7 & 8 & 3 & 0.0930 & $1.46 \times 10^{-21}$ & 2857 \\
\hline
\end{tabular}


It is obvious that $S(968717)$ is the lowest cost plan. Compared with other test plans, such as $S$ (968386) and $S$ (6765553), these test plans cannot satisfy either the risk constrains or the minimum cost constrain. Therefore, most importantly, when designing a reliability test plan, the designer needs to consider the test cost and related constrains, and find an optimum test plan.

\section{Conclusion}

In this research, a Bayesian reliability test design to the compound system, which consists of binomial subsystems, is conducted. To achieve this purpose, this paper presents the CSRTD methodology for verification of system reliability by means of information fusion and Monte Carlo method, and also the example of the missile launching system to demonstrate the proposed model. Comparing with current methods, the advantages of the proposed methods can be summarized: (1) the proposed model takes two types of prior information of the system $\left(\pi^{(1)}\left(R_{m+1}\right)\right.$ and $\left.\pi^{(2)}\left(R_{m+1}\right)\right)$ into consideration and completes two types of information fusion (in Section 2.2) on the basic of Bayesian theory and Monte Carlo method. Therefore, the posterior distribution of the compound system is figured out at last; (2) the proposed model focuses on the minimum cost of integral reliability test, so the test designers can find out an optimum test plan according to the totally cost.

\section{Reference}

[1] G. S., "Optimal Test Design for Reliability Demonstration," Operations Research, vol. 22, pp. 1236-1242, 1974.

[2] A. I. K., "The Design of Optimum Component Test Plans in the Demonstration of a Series System Reliability," Computational Statistics \& Data Analysis, vol. 14, pp. 281-292, Oct 1992.

[3] F. O. and e. al, "The design of optimum component test plans for system reliability," Computational Statistics \& Data Analysis, vol. 50, Jul 202006.

[4] A. Z. and e. al, "Bayesian reliability models of Weibull systems: State of the art," Appl. Math. Comput. Sci., vol. 22, pp. 585-600, 2012.

[5] S. Dey, "Bayesian Estimation of the Parameter and Reliability Function of an Inverse Rayleigh Distribution," Malaysian Journal of Mathematical Sciences, vol. 6, pp. 113-124, 2012.

[6] B. Cai, Y. Liu, Q. Fan, and Y. Zhang, "Application of Bayesian Networks to Reliabilit Evaluation of Software System for Subsea Blowout Preventers," International Journal of Control and Automation, vol. 6, pp. 47-59, 2013.

[7] M. S. Hamada, G. W. Alyson, and R. C. Shane, Byesian Reliability. New York: Springer, 2008.

[8] V. P. Savchuk and H. F. Martz, "Bayes Reliability Estimation Using Multiple Sources of Prior Information: Binomial Sampling," IEEE Transactions on Reliability, vol. 43, pp. 138-144, 1994. 